\title{
Subordination Properties of Multivalent Functions Defined by Generalized Multiplier Transformation
}

\author{
M. P. Jeyaraman ${ }^{1}$ and T. K. Suresh ${ }^{2}$ \\ ${ }^{1}$ Department of Mathematics, L. N. Government College, Ponneri, Chennai, Tamil Nadu 601 204, India \\ ${ }^{2}$ Department of Mathematics, Easwari Engineering College, Chennai, Tamil Nadu 600 089, India
}

Correspondence should be addressed to M. P. Jeyaraman; jeyaraman_mp@yahoo.co.in

Received 31 August 2013; Accepted 11 November 2013; Published 25 February 2014

Academic Editor: Janne Heittokangas

Copyright (C) 2014 M. P. Jeyaraman and T. K. Suresh. This is an open access article distributed under the Creative Commons Attribution License, which permits unrestricted use, distribution, and reproduction in any medium, provided the original work is properly cited.

\begin{abstract}
The main object of the present paper is to investigate several interesting subordination properties and a sharp inclusion relationship for certain subclass of multivalent analytic functions, which are defined here by the generalized multiplier transformation. Relevant connections of the results which are presented in this paper with various known results are also considered.
\end{abstract}

\section{Introduction and Definitions}

Let $\mathscr{A}$ denote the class of analytic functions in the open unit disk

$$
\mathbb{U}:=\{z: z \in \mathbb{C},|z|<1\}
$$

If $f$ and $g$ are in $\mathscr{A}$, we say that the function $f$ is said to be subordinate to $g$ or (equivalently) $g$ is said to be superordinate to $f$, written symbolically as

$$
f \prec g \quad \text { in } \mathbb{U} \quad \text { or } \quad f(z) \prec g(z) \quad(z \in \mathbb{U}),
$$

if there exists a Schwarz function $w$ analytic in $\mathbb{U}$, with $w(0)=$ 0 and $|w(z)|<1$, for all $z \in \mathbb{U}$, such that

$$
f(z)=g(w(z)) \quad(z \in \mathbb{U}) .
$$

In particular, if the function $g$ is univalent in $\mathbb{U}$, then we have the following equivalence (cf. $[1,2])$ :

$$
f \prec g \Longleftrightarrow f(0)=g(0), \quad f(\mathbb{U}) \subset g(\mathbb{U}) .
$$

Let $\mathscr{A}(p)(p \in \mathbb{N}=\{1,2, \ldots\})$ be the subclass of $\mathscr{A}$ consisting of functions $f$ defined by

$$
f(z)=z^{p}+\sum_{k=p+1}^{\infty} a_{k} z^{k} \quad(z \in \mathbb{U}),
$$

which are analytic and $p$-valent in the open unit disk $\mathbb{U}$. We note that $\mathscr{A}(1) \equiv \mathscr{A}$.

For a function $f \in \mathscr{A}(p)$ given by (5) and $g \in \mathscr{A}(p)$ defined by

$$
g(z)=z^{p}+\sum_{k=p+1}^{\infty} b_{k} z^{k}
$$

the Hadamard product (or convolution) of $f$ and $g$ is given by

$$
(f * g)(z):=z^{p}+\sum_{k=p+1}^{\infty} a_{k} b_{k} z^{k}=:(g * f)(z) .
$$

Recently, Cătaş [3] defined the generalized multiplier transformation $I_{p}^{m}(\lambda, l)$ on $\mathscr{A}(p)$ by the following infinite series:

$$
\begin{array}{r}
I_{p}^{m}(\lambda, l) f(z)=z^{p}+\sum_{k=p+1}^{\infty}\left(\frac{p+\lambda(k-p)+l}{p+l}\right)^{m} a_{k} z^{k} \\
\left(\lambda>0, l \geq 0, p \in \mathbb{N}, m \in \mathbb{N}_{0}=\mathbb{N} \cup\{0\}, z \in \mathbb{U}\right) .
\end{array}
$$

We note that

$$
I_{p}^{0}(1,0) f(z)=f(z), \quad I_{p}^{1}(1,0) f(z)=\frac{z f^{\prime}(z)}{p} .
$$


It is easily verified from (8) that

$$
\begin{aligned}
& \lambda z\left(I_{p}^{m}(\lambda, l) f\right)^{\prime}(z) \\
& \quad=(p+l) I_{p}^{m+1}(\lambda, l) f(z)-[p(1-\lambda)+l] I_{p}^{m}(\lambda, l) f(z) .
\end{aligned}
$$

The generalized multiplier transformation $I_{p}^{m}(\lambda, l)$ reduces several familiar operators by specializing the parameters $m, \lambda, l$, and $p$.

(1) For the choice of $\lambda=1$, the operator defined by (8) reduces the operator $I_{p}(m, l)$, studied by Srivastava et al. [4] and Sivaprasad Kumar et al. [5].

(2) By taking $\lambda=p=1$, the generalized multiplier transformation $I_{p}^{m}(\lambda, l)$ yields the operator $I_{l}^{m}$, which was investigated by Cho et al. $[6,7]$.

(3) For $\lambda=1$ and $l=0$, the operator $I_{p}^{m}(\lambda, l)$ reduces the differential operator $D_{p}^{m}$ studied by Kamali and Orhan [8] and Orhan and Kiziltunç [9] and also, for $\lambda=p=1$ and $l=0$, it yields the differential operator $D^{m}$ introduced by Sălăgean [10].

(4) As a special case of this operator $I_{p}^{m}(\lambda, l)$ for $l=0$ and $p=1$, it reduces the generalized Sălăgean operator $D_{\lambda}^{m}$ studied by Al-Oboudi [11] and also earlier, for $\lambda=$ $l=p=1$, it gives the operator $I^{m}$ investigated by Uralegaddi and Somanatha [12].

Now, we introduce a new subclass of functions in $\mathscr{A}(p)$, by making use of the generalized multiplier transformation $I_{p}^{m}(\lambda, l)$ as follows.

Definition 1. Let $m \in \mathbb{N}_{0}, A, B, \alpha, \lambda, l$ be arbitrary fixed real numbers such that $-1 \leq B<A \leq 1,0 \leq \alpha<p, \lambda>0, p \in \mathbb{N}$, and $l \geq 0$. A function $f \in \mathscr{A}(p)$ is said to be in the class $\mathscr{R}_{p}^{m}(\lambda, l, \alpha ; A, B)$, if it satisfies the following subordination condition:

$$
\frac{1}{p-\alpha}\left(\frac{z\left(I_{p}^{m}(\lambda, l) f\right)^{\prime}(z)}{I_{p}^{m}(\lambda, l) f(z)}-\alpha\right) \prec \frac{1+A z}{1+B z}, \quad(z \in \mathbb{U}) .
$$

In particular, for $A=1$ and $B=-1$, we write $\mathscr{R}_{p}^{m}(\lambda, l, \alpha$; $1,-1)=\mathscr{R}_{p}^{m}(\lambda, l, \alpha)$, where

$$
\begin{aligned}
& \mathscr{R}_{p}^{m}(\lambda, l, \alpha) \\
& \quad=\left\{f \in \mathscr{A}(p): \operatorname{Re}\left(\frac{z\left(I_{p}^{m}(\lambda, l) f\right)^{\prime}(z)}{I_{p}^{m}(\lambda, l) f(z)}\right)>\alpha, z \in \mathbb{U}\right\} .
\end{aligned}
$$

Motivated by the recent work of Bulboacă et al. [13] and Patel and Mishra [14], we investigate the subordination properties of the generalized multiplier transformation $I_{p}^{m}(\lambda, l)$ defined by (8) and obtain a sharp inclusion relationship for the multivalent analytic function class $\mathscr{R}_{p}^{m}(\lambda, l, \alpha ; A, B)$. We also derive a number of sufficient conditions for functions belonging to the subclass $\mathscr{R}_{p}^{m}(\lambda, l, \alpha)$ which satisfy certain subordination properties. Relevant connections of the results presented in this paper with earlier sequels are also pointed out.

\section{Preliminaries}

To prove our results, we will need the following lemmas.

Lemma 2 (see $[1,2])$. Let a function $h$ be analytic and convex (univalent) in $\mathbb{U}$, with $h(0)=1$. Suppose also that the function $\varphi$ given by

$$
\varphi(z)=1+b_{1} z+b_{2} z^{2}+\cdots
$$

is analytic in $\mathbb{U}$. If

$$
\varphi(z)+\frac{z \varphi^{\prime}(z)}{c} \prec h(z) \quad(\operatorname{Re} c \geq 0, c \neq 0),
$$

then

$$
\varphi(z) \prec \psi(z)=\frac{c}{z^{c}} \int_{0}^{z} t^{c-1} h(t) d t \prec h(z)
$$

where $\psi$ is the best dominant of (14).

We denote by $P(\gamma)$ the class of functions $\varphi$ given by (13) which are analytic in $\mathbb{U}$ and satisfy the following inequality:

$$
\operatorname{Re} \varphi(z)>\gamma, \quad(0 \leq \gamma<1, z \in \mathbb{U})
$$

Lemma 3 (see [15]). Let the function $\varphi$ given by (13) be in the class $P(\gamma)$. Then

$$
\operatorname{Re} \varphi(z) \geq 2 \gamma-1+\frac{2(1-\gamma)}{1+|z|} \quad(0 \leq \gamma<1, z \in \mathbb{U}) .
$$

Lemma 4 (see [16]). For $0 \leq \gamma_{1}<\gamma_{2}<1$,

$$
\begin{gathered}
P\left(\gamma_{1}\right) * P\left(\gamma_{2}\right) \subset P\left(\gamma_{3}\right), \\
\text { where } \gamma_{3}=1-2\left(1-\gamma_{1}\right)\left(1-\gamma_{2}\right) .
\end{gathered}
$$

The result is the best possible.

For any complex numbers $a, b$, and $c\left(c \notin \mathbb{Z}_{0}^{-}:=\{0,-1\right.$, $-2, \ldots\})$, the Gaussian hypergeometric function is defined by

$$
{ }_{2} F_{1}(a, b ; c ; z)=1+\frac{a b}{c} \frac{z}{1 !}+\frac{a(a+1) b(b+1)}{c(c+1)} \frac{z^{2}}{2 !}+\cdots .
$$


Lemma 5 (see [17]). For any complex numbers $a, b, c\left(c \notin \mathbb{Z}_{0}^{-}\right)$, one has

$$
\begin{gathered}
\int_{0}^{1} t^{b-1}(1-t)^{c-b-1}(1-z t)^{-a} d t \\
=\frac{\Gamma(b) \Gamma(c-b)}{\Gamma(c)}{ }_{2} F_{1}(a, b ; c ; z), \\
\operatorname{Re} c>\operatorname{Re} b>0, \\
{ }_{2} F_{1}(a, b ; c ; z)=(1-z)^{-a}{ }_{2} F_{1}\left(a, c-b ; c ; \frac{z}{z-1}\right), \\
{ }_{2} F_{1}(a, b ; c ; z)={ }_{2} F_{1}(b, a ; c ; z), \\
(b+1){ }_{2} F_{1}(1, b ; b+1 ; z)=(b+1)+b z{ }_{2} F_{1}(1, b+1 ; b+2 ; z) .
\end{gathered}
$$

Lemma 6 (see [18]). If $-1 \leq B<A \leq 1, \beta>0$, and the complex number $\gamma$ is constrained by $\operatorname{Re}(\gamma) \geq-\beta(1-A) /(1-B)$, then the following differential equation:

$$
q(z)+\frac{z q^{\prime}(z)}{\beta q(z)+\gamma}=\frac{1+A z}{1+B z} \quad(z \in \mathbb{U})
$$

has a univalent solution in $\mathbb{U}$ given by

$$
q(z)= \begin{cases}\frac{z^{\beta+\gamma}(1+B z)^{\beta(A-B) / B}}{\beta \int_{0}^{z} t^{\beta+\gamma-1}(1+B t)^{\beta(A-B) / B} d t}-\frac{\gamma}{\beta}, & \text { if } B \neq 0, \\ \frac{z^{\beta+\gamma} \exp (\beta A z)}{\beta \int_{0}^{z} t^{\beta+\gamma-1} \exp (\beta A t) d t}-\frac{\gamma}{\beta}, & \text { if } B=0 .\end{cases}
$$

If the function $\varphi(z)$ given by $(13)$ is analytic in $\mathbb{U}$ and satisfies the following subordination:

$$
\varphi(z)+\frac{z \varphi^{\prime}(z)}{\beta \varphi(z)+\gamma} \prec \frac{1+A z}{1+B z} \quad(z \in \mathbb{U}),
$$

then

$$
\varphi(z) \prec q(z) \prec \frac{1+A z}{1+B z} \quad(z \in \mathbb{U}),
$$

and $q(z)$ is the best dominant of (23).

Lemma 7 (see [19]). Let $v$ be a positive measure on the interval $[0,1]$. Let $h(z, t)$ be a complex-valued function defined on $\mathbb{U} \times[0,1]$ such that $h(\cdot, t)$ is analytic in $\mathbb{U}$ for each $t \in[0,1]$ and $h(z, \cdot)$ is $v$-integrable on $[0,1]$ for each $z \in \mathbb{U}$. In addition, suppose that $\operatorname{Re}(h(z, t))>0, h(-r, t)$ is real, and

$$
\operatorname{Re}\left(\frac{1}{h(z, t)}\right) \geq \frac{1}{h(-r, t)} \quad(|z| \leq r<1 ; t \in[0,1]) .
$$

If the function $\mathrm{H}(z)$ is defined by

$$
H(z)=\int_{0}^{1} h(z, t) d v(t)
$$

then

$$
\operatorname{Re}\left(\frac{1}{H(z)}\right) \geq \frac{1}{H(-r)} \quad(|z| \leq r<1) .
$$

Lemma 8 (see [20]). Let $\lambda \neq 0$ be a real number, $a / \lambda>0$, and $0 \leq \beta<1$. Let $g(z)=1+c_{n} z^{n}+c_{n+1} z^{n+1}+\cdots$ be analytic in $\mathbb{U}$ and

$$
g(z) \prec 1+\frac{a M z}{n \lambda+a} \quad(n \in \mathbb{N}),
$$

where

$$
M=\frac{(1-\beta)|\lambda|(1+n \lambda / a)}{|1-\lambda+\lambda \beta|+\sqrt{1+(1+n \lambda / a)^{2}}} .
$$

If $P(z)=1+d_{n} z^{n}+d_{n+1} z^{n+1}+\cdots$ is analytic in $\mathbb{U}$ and satisfies the subordination relation

$$
g(z)\{1-\lambda+\lambda[(1-\beta) P(z)+\beta]\} \prec 1+M z,
$$

then $\operatorname{Re} P(z)>0$ for $z \in \mathbb{U}$.

Lemma 9 (see [2]). Suppose that the function $\Psi: \mathbb{C}^{2} \times \mathbb{U} \rightarrow$ $\mathbb{C}$ satisfies the following condition:

$$
\operatorname{Re} \Psi(i x, y ; z) \leq \varepsilon
$$

for all $x \in \mathbb{R}$ and $y \leq-(1 / 2)\left(1+x^{2}\right)$ and for all $z \in \mathbb{U}$. If the function $\varphi$ of the form (13) is analytic in $\mathbb{U}$ and

$$
\operatorname{Re} \Psi\left(\varphi(z), z \varphi^{\prime}(z) ; z\right)>\varepsilon
$$

then

$$
\operatorname{Re}(\varphi(z))>0 \quad(z \in \mathbb{U})
$$

\section{Subordination Properties of $I_{p}^{m}(\lambda, l)$}

Unless otherwise mentioned, we assume throughout this paper that

$$
\begin{gathered}
\lambda>0, \quad l \geq 0, \quad m \in \mathbb{N}_{0}, \quad 0 \leq \alpha<p, \quad p \in \mathbb{N}, \\
-1 \leq B<A \leq 1 .
\end{gathered}
$$

Theorem 10. Let $\beta>0$ and $-1 \leq B_{j}<A_{j} \leq 1, j=1,2$. If the functions $f_{j} \in \mathscr{A}(p)$ satisfy the following subordination condition:

$$
\begin{array}{r}
(1-\beta) \frac{I_{p}^{m}(\lambda, l) f_{j}(z)}{z^{p}}+\beta \frac{I_{p}^{m+1}(\lambda, l) f_{j}(z)}{z^{p}} \prec \frac{1+A_{j} z}{1+B_{j} z}, \\
j=1,2,
\end{array}
$$

then

$$
(1-\beta) \frac{I_{p}^{m}(\lambda, l) F(z)}{z^{p}}+\beta \frac{I_{p}^{m+1}(\lambda, l) F(z)}{z^{p}} \prec \frac{1+(1-2 \delta) z}{1-z},
$$


where $F=I_{p}^{m}(\lambda, l)\left(f_{1} * f_{2}\right)$ and

$$
\begin{aligned}
\delta= & 1-\frac{4\left(A_{1}-B_{1}\right)\left(A_{2}-B_{2}\right)}{\left(1-B_{1}\right)\left(1-B_{2}\right)} \\
& \times\left(1-\frac{1}{2}{ }_{2} F_{1}\left(1,1 ; \frac{p+l}{\lambda \beta}+1 ; \frac{1}{2}\right)\right) .
\end{aligned}
$$

The result is the best possible when $B_{1}=B_{2}=-1$.

Proof. Let the functions $f_{j} \in \mathscr{A}(p), j=1,2$, satisfy the subordination condition (35). Then, by setting

$$
\begin{aligned}
\varphi_{j}(z) & =(1-\beta) \frac{I_{p}^{m}(\lambda, l) f_{j}(z)}{z^{p}}+\beta \frac{I_{p}^{m+1}(\lambda, l) f_{j}(z)}{z^{p}} \\
& \prec \frac{1+A_{j} z}{1+B_{j} z}, \quad j=1,2,
\end{aligned}
$$

we have

$$
\varphi_{j} \in P\left(\gamma_{j}\right), \quad \gamma_{j}=\frac{1-A_{j}}{1-B_{j}}, \quad j=1,2
$$

By making use of (10) and (38), we obtain

$$
\begin{array}{r}
I_{p}^{m}(\lambda, l) f_{j}(z)=\frac{p+l}{\lambda \beta} z^{p-(p+l) / \lambda \beta} \int_{0}^{z} t^{(p+l) / \lambda \beta-1} \varphi_{j}(t) d t \\
j=1,2 .
\end{array}
$$

Now, if we let $F=I_{p}^{m}(\lambda, l)\left(f_{1} * f_{2}\right)$, then by using (40) and the fact that

$$
\begin{aligned}
I_{p}^{m}(\lambda, l) F(z) & =I_{p}^{m}(\lambda, l)\left(I_{p}^{m}(\lambda, l)\left(f_{1} * f_{2}\right)(z)\right) \\
& =I_{p}^{m}(\lambda, l) f_{1}(z) * I_{p}^{m}(\lambda, l) f_{2}(z),
\end{aligned}
$$

a simple computation shows that

$$
I_{p}^{m}(\lambda, l) F(z)=\frac{p+l}{\lambda \beta} z^{p-(p+l) / \lambda \beta} \int_{0}^{z} t^{(p+l) / \lambda \beta-1} \varphi_{0}(t) d t
$$

where

$$
\begin{aligned}
\varphi_{0}(z) & =(1-\beta) \frac{I_{p}^{m}(\lambda, l) F(z)}{z^{p}}+\beta \frac{I_{p}^{m+1}(\lambda, l) F(z)}{z^{p}} \\
& =\frac{p+l}{\lambda \beta} z^{-(p+l) / \lambda \beta} \int_{0}^{z} t^{(p+l) / \lambda \beta-1}\left(\varphi_{1} * \varphi_{2}\right)(t) d t .
\end{aligned}
$$

Since $\varphi_{j} \in P\left(\gamma_{j}\right), j=1,2$, it follows from Lemma 4 that

$$
\varphi_{1} * \varphi_{2} \in P\left(\gamma_{3}\right), \quad \text { where } \gamma_{3}=1-2\left(1-\gamma_{1}\right)\left(1-\gamma_{2}\right) \text {, }
$$

and the bound $\gamma_{3}$ is the best possible. Hence, by using Lemma 3 in (43), we deduce that

$$
\begin{aligned}
\operatorname{Re} \varphi_{0}(z) & \frac{p+l}{\lambda \beta} \int_{0}^{1} u^{(p+l) / \lambda \beta-1} \operatorname{Re}\left(\varphi_{1} * \varphi_{2}\right)(u z) d u \\
\geq & \frac{p+l}{\lambda \beta} \int_{0}^{1} u^{(p+l) / \lambda \beta-1}\left(2 \gamma_{3}-1+\frac{2\left(1-\gamma_{3}\right)}{1+u|z|}\right) d u \\
> & \frac{p+l}{\lambda \beta} \int_{0}^{1} u^{(p+l) / \lambda \beta-1}\left(2 \gamma_{3}-1+\frac{2\left(1-\gamma_{3}\right)}{1+u}\right) d u \\
= & 1-\frac{4\left(A_{1}-B_{1}\right)\left(A_{2}-B_{2}\right)}{\left(1-B_{1}\right)\left(1-B_{2}\right)} \\
& \times\left(1-\frac{p+l}{\lambda \beta} \int_{0}^{1} \frac{u^{(p+l) / \lambda \beta-1}}{1+u} d u\right)=\delta \quad(z \in \mathbb{U}),
\end{aligned}
$$

where $\delta$ is given by (37).

When $B_{1}=B_{2}=-1$, we consider the functions $f_{j} \in$ $\mathscr{A}(p)(j=1,2)$ which satisfy the hypothesis (35) and are given by

$$
\begin{array}{r}
I_{p}^{m}(\lambda, l) f_{j}(z)=\frac{p+l}{\lambda \beta} z^{p-(p+l) / \lambda \beta} \int_{0}^{z} t^{(p+l) / \lambda \beta-1}\left(\frac{1+A_{j} t}{1-t}\right) d t \\
j=1,2 .
\end{array}
$$

Since

$$
\begin{aligned}
& \left(\frac{1+A_{1} z}{1-z}\right) *\left(\frac{1+A_{2} z}{1-z}\right) \\
& \quad=1-\left(1+A_{1}\right)\left(1+A_{2}\right)+\frac{\left(1+A_{1}\right)\left(1+A_{2}\right)}{1-z}
\end{aligned}
$$

it follows from (43) that

$$
\begin{aligned}
\varphi_{0}(z)= & \frac{p+l}{\lambda \beta} \\
& \times \int_{0}^{1} u^{(p+l) / \lambda \beta-1}\left(1-\left(1+A_{1}\right)\left(1+A_{2}\right)\right. \\
& \left.\quad+\frac{\left(1+A_{1}\right)\left(1+A_{2}\right)}{1-u z}\right) d u \\
= & 1-\left(1+A_{1}\right)\left(1+A_{2}\right) \\
& +\frac{\left(1+A_{1}\right)\left(1+A_{2}\right)}{(1-z)}{ }_{2} F_{1}\left(1,1 ; \frac{p+l}{\lambda \beta}+1 ; \frac{z}{z-1}\right) .
\end{aligned}
$$


Therefore

$$
\begin{aligned}
\varphi_{0}(z) \longrightarrow & 1-\left(1+A_{1}\right)\left(1+A_{2}\right) \\
& +\frac{1}{2}\left(1+A_{1}\right)\left(1+A_{2}\right) \\
& \times{ }_{2} F_{1}\left(1,1 ; \frac{p+l}{\lambda \beta}+1 ; \frac{1}{2}\right) \text { as } z \longrightarrow-1,
\end{aligned}
$$

which evidently completes our proof of Theorem 10 .

By setting $\beta=1, B_{j}=-1$, and $A_{j}=1-2 \delta_{j}, j=1,2$, in Theorem 10, we have the following corollary.

Corollary 11. If the functions $f_{j} \in \mathscr{A}(p)$ satisfy the following subordination condition

$$
\frac{I_{p}^{m+1}(\lambda, l) f_{j}(z)}{z^{p}} \prec \frac{1+\left(1-2 \delta_{j}\right) z}{1-z}, \quad j=1,2,
$$

then

$$
\begin{aligned}
\operatorname{Re}\left(\frac{I_{p}^{m+1}(\lambda, l) F(z)}{z^{p}}\right) \\
>1-2\left(1-\delta_{1}\right)\left(1-\delta_{2}\right) \\
\quad \times\left[2-{ }_{2} F_{1}\left(1,1 ; \frac{p+l}{\lambda}+1 ; \frac{1}{2}\right)\right] \quad(z \in \mathbb{U}),
\end{aligned}
$$

where $F=I_{p}^{m}(\lambda, l)\left(f_{1} * f_{2}\right)$.

In Theorem 12, we have determined the sufficient condition for the functions $I_{p}^{m}(\lambda, l) f(z) / z^{p}$ to be a member of the class $P(\eta)$.

Theorem 12. If $f \in \mathscr{A}(p)$ satisfy the following subordination condition:

$$
(1-\beta) \frac{I_{p}^{m}(\lambda, l) f(z)}{z^{p}}+\beta \frac{I_{p}^{m+1}(\lambda, l) f(z)}{z^{p}} \prec \frac{1+A z}{1+B z},
$$

then

$$
\operatorname{Re}\left(\frac{I_{p}^{m}(\lambda, l) f(z)}{z^{p}}\right)>\eta \quad(z \in \mathbb{U})
$$

where

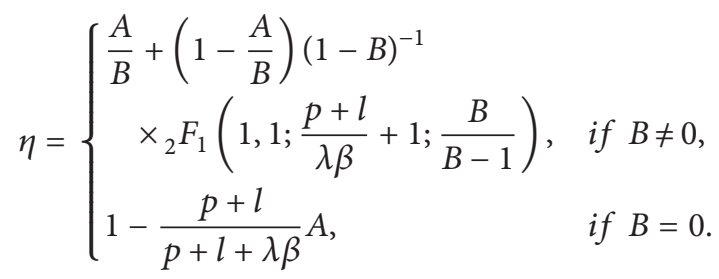

The result is the best possible.

Proof. Let

$$
g(z)=\frac{I_{p}^{m}(\lambda, l) f(z)}{z^{p}} \quad \text { for } f \in \mathscr{A}(p) .
$$

Then, the function $g$ is of the form (13). Differentiating (55) with respect to $z$ and using the identity (10), we obtain

$$
\frac{I_{p}^{m+1}(\lambda, l) f(z)}{z^{p}}=g(z)+\frac{\lambda}{p+l} z g^{\prime}(z) .
$$

By using (52), (55), and (56), we get

$$
g(z)+\frac{\lambda \beta}{p+l} z g^{\prime}(z) \prec \frac{1+A z}{1+B z} .
$$

Now, by applying Lemma 2, we have

$$
g(z) \prec Q(z)=\frac{p+l}{\lambda \beta} z^{-(p+l) / \lambda \beta} \int_{0}^{z} t^{(p+l) / \lambda \beta-1}\left(\frac{1+A t}{1+B t}\right) d t .
$$

By using Lemma 5, we get

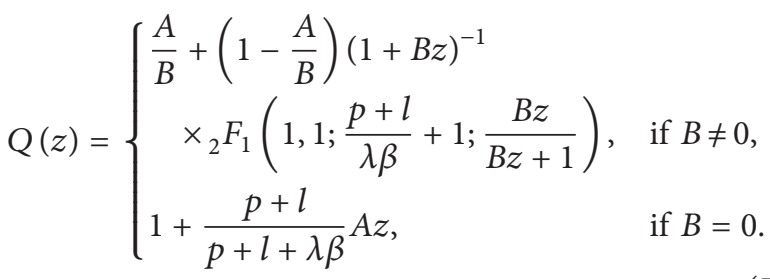

Now, we will show that

$$
\inf \{\operatorname{Re} Q(z):|z|<1\}=Q(-1) .
$$

We have

$$
\operatorname{Re} \frac{1+A z}{1+B z} \geq \frac{1-A r}{1-B r} \quad|z|=r<1
$$

and setting

$$
\begin{gathered}
h(s, z)=\frac{1+A z s}{1+B z s} \quad(0 \leq s \leq 1), \\
d \mu(s)=\frac{p+l}{\lambda \beta} s^{(p+l) / \lambda \beta} d s,
\end{gathered}
$$

which is a positive measure on the closed interval $[0,1]$, we get

$$
Q(z)=\int_{0}^{1} h(s, z) d \mu(s)
$$

so that

$$
\operatorname{Re} Q(z) \geq \int_{0}^{1} \frac{1-A s r}{1-B s r} d \mu(s)=Q(-r), \quad|z|=r<1 .
$$

As $r \rightarrow 1^{-}$in (64), we obtain the assertion (60). Now, by using (59) and (60), we get

$$
\operatorname{Re}\left(\frac{I_{p}^{m}(\lambda, l) f(z)}{z^{p}}\right)>\eta
$$

where $\eta$ is given by (54). 
To show that the estimate (54) is the best possible, we consider the function $f \in \mathscr{A}(p)$ defined by

$$
\frac{I_{p}^{m}(\lambda, l) f(z)}{z^{p}}=\frac{p+l}{\lambda \beta} \int_{0}^{1} u^{(p+l) / \lambda \beta-1}\left(\frac{1+A u z}{1+B u z}\right) d u .
$$

For the above function, we find that

$$
\begin{aligned}
& (1-\beta) \frac{I_{p}^{m}(\lambda, l) f(z)}{z^{p}}+\beta \frac{I_{p}^{m+1}(\lambda, l) f(z)}{z^{p}}=\frac{1+A z}{1+B z}, \\
& \frac{I_{p}^{m}(\lambda, l) f(z)}{z^{p}} \\
& \longrightarrow \frac{p+l}{\lambda \beta} \int_{0}^{1} u^{(p+l) / \lambda \beta-1}\left(\frac{1-A u}{1-B u}\right) d u
\end{aligned}
$$

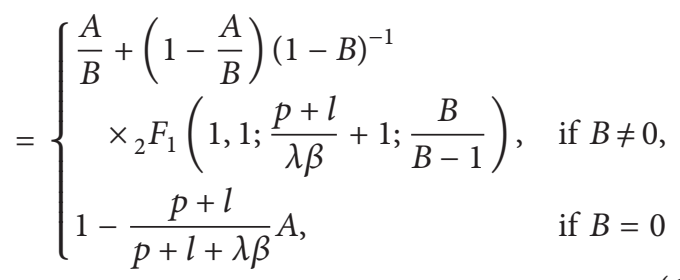

as $z \rightarrow-1$, and the proof of the Theorem 12 is completed.

In its special case when $A=1-2 \gamma, B=-1$ and $\beta=1$, Theorem 12 yields the following corollary.

Corollary 13. If $f \in \mathscr{A}(p)$ satisfy the following condition:

$$
\frac{I_{p}^{m+1}(\lambda, l) f(z)}{z^{p}} \prec \frac{1+(1-2 \gamma) z}{1-z} \quad(z \in \mathbb{U}),
$$

then

$$
\begin{aligned}
\operatorname{Re}\left(\frac{I_{p}^{m}(\lambda, l) f(z)}{z^{p}}\right) \\
>\gamma+(1-\gamma) \\
\quad \times\left[{ }_{2} F_{1}\left(1,1 ; \frac{p+l}{\lambda}+1 ; \frac{1}{2}\right)-1\right] \quad(z \in \mathbb{U}) .
\end{aligned}
$$

The result is the best possible.

For a function $f \in \mathscr{A}(p)$, the integral operator

$$
\begin{aligned}
F_{\mu, p} f(z)= & \frac{\mu+p}{z^{\mu}} \int_{0}^{z} t^{\mu-1} f(t) d t \\
= & z^{p}{ }_{2} F_{1}(1, \mu+p ; \mu+p+1 ; z) * f(z) \\
& (\mu>-p, z \in \mathbb{U}) .
\end{aligned}
$$

Also, it is easily verified from (70) that

$$
\begin{aligned}
z\left(I_{p}^{m}\right. & \left.(\lambda, l) F_{\mu, p} f\right)^{\prime}(z) \\
& =(\mu+p) I_{p}^{m}(\lambda, l) f(z)-\mu I_{p}^{m}(\lambda, l) F_{\mu, p} f(z) .
\end{aligned}
$$

In the next Theorem 14, by using the integral operator defined in (70), we established the sufficient condition for the functions $I_{p}^{m}(\lambda, l) F_{\mu, p} f(z) / z^{p}$ belongs to $P\left(\rho_{0}\right)$.

Theorem 14. If $f \in \mathscr{A}(p)$ and $F_{\mu, p} f$ is given by (70), satifies the subordination condition:

$$
(1-\beta) \frac{I_{p}^{m}(\lambda, l) F_{\mu, p} f(z)}{z^{p}}+\beta \frac{I_{p}^{m}(\lambda, l) f(z)}{z^{p}} \prec \frac{1+A z}{1+B z},
$$

then

$$
\operatorname{Re}\left(\frac{I_{p}^{m}(\lambda, l) F_{\mu, p} f(z)}{z^{p}}\right)>\rho_{0} \quad(z \in \mathbb{U})
$$

where

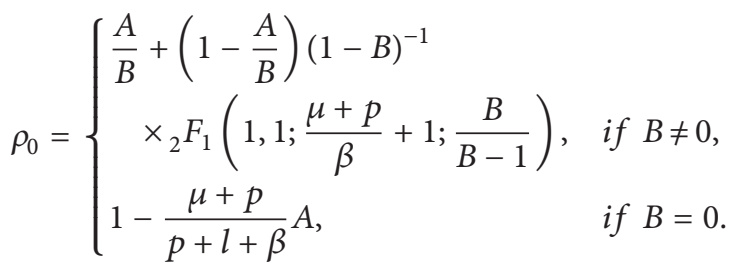

The result is the best possible.

Proof. Let

$$
h(z)=\frac{I_{p}^{m}(\lambda, l) F_{\mu, p} f(z)}{z^{p}} .
$$

Then by using the hypothesis (72) together with (71) and (75), we obtain

$$
\begin{aligned}
& h(z)+\frac{\beta}{\mu+p} z h^{\prime}(z) \\
& \quad=(1-\beta) \frac{I_{p}^{m}(\lambda, l) F_{\mu, p} f(z)}{z^{p}}+\beta \frac{I_{p}^{m}(\lambda, l) f(z)}{z^{p}} \prec \frac{1+A z}{1+B z} .
\end{aligned}
$$

The remaining part of the proof of Theorem 14 is similar to that of Theorem 12 and hence we omit the details.

\section{Inclusion Relationship for the Class $\mathscr{R}_{p}^{m}(\lambda, l, \alpha ; A, B)$}

Theorem 15. If $f(z) \in \mathscr{R}_{p}^{m+1}(\lambda, l, \alpha ; A, B)$ and

$$
\lambda(p-\alpha)(1-A)+[\alpha \lambda+p(1-\lambda)+l](1-B) \geq 0
$$

then

$$
\frac{1}{p-\alpha}\left(\frac{z\left(I_{p}^{m}(\lambda, l) f\right)^{\prime}(z)}{I_{p}^{m}(\lambda, l) f(z)}-\alpha\right) \prec q(z) \prec \frac{1+A z}{1+B z}
$$

$$
(z \in \mathbb{U})
$$


where

$$
\begin{gathered}
q(z)=\frac{1}{\lambda(p-\alpha)}\left(\frac{1}{Q(z)}-[\alpha \lambda+p(1-\lambda)+l]\right), \\
Q(z)= \begin{cases}\int_{0}^{1} t^{p+l-1}\left(\frac{1+B t z}{1+B z}\right)^{\lambda(p-\alpha)(A-B) / B} d t, & \text { if } B \neq 0, \\
\int_{0}^{1} t^{p+l-1} \exp (\lambda(p-\alpha) A z(t-1)) d t, & \text { if } B=0\end{cases}
\end{gathered}
$$

and $q(z)$ is the best dominant of (78). If, in addition to (77),

$$
A>\frac{-B[\alpha \lambda+p(1-\lambda)+l+1]}{\lambda(p-\alpha)} \text { with }-1 \leq B<0 \text {, }
$$

then

$$
\mathscr{R}_{p}^{m+1}(\lambda, l, \alpha ; A, B) \subset \mathscr{R}_{p}^{m}(\lambda, l, \alpha ; 1-2 \rho,-1),
$$

where

$$
\begin{aligned}
\rho= & \frac{1}{\lambda(p-\alpha)} \\
& \times((p+l) \\
& \times\left[{ }_{2} F_{1}\left(1, \frac{\lambda(p-\alpha)(B-A)}{B} ; p+l+1 ; \frac{B}{B-1}\right)\right]^{-1} \\
& \quad-[\alpha \lambda+p(1-\lambda)+l]) .
\end{aligned}
$$

The bound on $\rho$ is the best possible.

Proof. Let $f(z) \in \mathscr{R}_{p}^{m+1}(\lambda, l, \alpha ; A, B)$. Define the function $g$ by

$$
g(z)=z\left(\frac{I_{p}^{m}(\lambda, l) f(z)}{z^{p}}\right)^{1 /(p-\alpha)}
$$

and $r_{1}=\sup \{r: g(z) \neq 0,0<|z|<r<1\}$. Then $g(z)$ is single-valued and analytic function in $|z|<r_{1}$. By logarithmic differentiation in (83), it follows that the function $\phi(z)$ given by

$$
\begin{aligned}
\phi(z) & =\frac{z g^{\prime}(z)}{g(z)}=\frac{1}{p-\alpha}\left(\frac{z\left(I_{p}^{m}(\lambda, l) f\right)^{\prime}(z)}{I_{p}^{m}(\lambda, l) f(z)}-\alpha\right) \\
& \prec \frac{1+A z}{1+B z}
\end{aligned}
$$

is analytic in $|z|<r_{1}$ and $\phi(0)=1$. Using the identity (10) in (84) and logarithmic differentiation of the resulting equation yields the following:

$$
\begin{aligned}
\frac{1}{p-\alpha} & \left(\frac{z\left(I_{p}^{m+1}(\lambda, l) f\right)^{\prime}(z)}{I_{p}^{m+1}(\lambda, l) f(z)}-\alpha\right) \\
& =\phi(z)+\frac{\lambda z \phi^{\prime}(z)}{\lambda(p-\alpha) \phi(z)+\alpha \lambda+p(1-\lambda)+l} \\
& \prec \frac{1+A z}{1+B z} \quad\left(|z|<r_{1}\right) .
\end{aligned}
$$

Hence, by using Lemma 6 with $\beta=\lambda(p-\alpha)$ and $\gamma=\alpha \lambda+$ $p(1-\lambda)+l$, we find that

$$
\begin{aligned}
\phi(z) & \prec \frac{1}{\lambda(p-\alpha)}\left(\frac{1}{Q(z)}-[\alpha \lambda+p(1-\lambda)+l]\right) \\
& =q(z) \prec \frac{1+A z}{1+B z} \quad\left(|z|<r_{1}\right),
\end{aligned}
$$

where $q(z)$ is the best dominant of $(86)$ and $Q(z)$ is given by (79). Since

$$
\operatorname{Re}\left(\frac{1+A z}{1+B z}\right)>0 \quad(-1 \leq B<A \leq 1 ; z \in \mathbb{U}),
$$

by (86), we have $\operatorname{Re}(\phi(z))>0\left(|z|<r_{1}\right)$. Now, (84) shows that $g(z)$ is starlike (univalent) in $|z|<r_{1}$. Thus, it is not possible that $g(z)$ vanishes on $|z|=r_{1}$ if $r_{1}<1$. So, we conclude that $r_{1}=1$, and, therefore, $\phi(z)$ is analytic in $\mathbb{U}$. Hence, (86) implies that

$$
\phi(z) \prec q(z) \prec \frac{1+A z}{1+B z} \quad(z \in \mathbb{U}) .
$$

This proves the assertion (78) of Theorem 15.

In order to establish (81), we have to find the greatest lower bound of $\rho(0<\rho<1)$ such that

$$
\phi(z) \prec \frac{1+(1-2 \rho) z}{1-z} \quad(z \in \mathbb{U}) .
$$

By (86), we have to show that

$$
\rho=\inf _{z \in \mathbb{U}} \operatorname{Re}(q(z))=q(-1) .
$$

To prove (90), we need to show that

$$
\inf _{z \in \mathbb{U}} \operatorname{Re}\left(\frac{1}{Q(z)}\right)=\frac{1}{Q(-1)} .
$$

From (79), we see that, for $B \neq 0$,

$$
Q(z)=(1+B z)^{a} \int_{0}^{1} t^{b-1}(1-t)^{c-b-1}(1+B z t)^{-a} d t
$$

$(z \in \mathbb{U})$

where

$$
a=\frac{\lambda(p-\alpha)(B-A)}{B}, \quad b=p+l, \quad c=p+l+1 .
$$


Since $c>b>0$, by using Lemma 5 , we get following:

$$
\begin{aligned}
Q(z) & =(1+B z) \frac{\Gamma(b) \Gamma(c-b)}{\Gamma(c)}{ }_{2} F_{1}(a, b ; c ;-B z) \\
& =\frac{\Gamma(b)}{\Gamma(c)}{ }_{2} F_{1}\left(a, c-b ; c ; \frac{B z}{B z+1}\right) \\
& =\frac{\Gamma(b)}{\Gamma(c)}{ }_{2} F_{1}\left(1, a ; c ; \frac{B z}{B z+1}\right) .
\end{aligned}
$$

Since

$$
A>\frac{-B[\alpha \lambda+p(1-\lambda)+l+1]}{\lambda(p-\alpha)} \text { with }-1 \leq B<0
$$

implies that $c>a>0$, by using Lemma 5, we find from (94) that

$$
Q(z)=\int_{0}^{1} h(z, t) d v(t)
$$

where

$$
\begin{gathered}
h(z, t)=\frac{1+B z}{1+(1-t) B z} \quad(0 \leq t \leq 1), \\
d v(t)=\frac{\Gamma(b)}{\Gamma(a) \Gamma(c-a)} t^{a-1}(1-t)^{c-a-1} d t,
\end{gathered}
$$

which is positive measure on $[0,1]$. For $-1 \leq B<0$, it may be noted that $\operatorname{Re}(h(z, t))>0$ and $h(-r, t)$ is real for $0 \leq|z| \leq$ $r<1$ and $t \in[0,1]$. Hence, by using Lemma 7 , we have

$$
\begin{gathered}
\operatorname{Re}\left(\frac{1}{Q(z)}\right) \geq \frac{1}{Q(-r)} \quad(|z| \leq r<1), \\
\inf _{z \in \mathbb{U}} \operatorname{Re}\left(\frac{1}{Q(z)}\right)=\inf _{-1<r<1} \frac{1}{Q(-r)}=\frac{1}{\int_{0}^{1} h(-1, t) d v(t)} \\
=\frac{1}{Q(-1)} .
\end{gathered}
$$

We note that $Q(-1) \neq 0$. Thus, by using (90) and (94), we have

$$
\begin{aligned}
& \rho=\frac{1}{\lambda(p-\alpha)} \\
& \quad \times((p+l) \\
& \quad \times\left[{ }_{2} F_{1}\left(1, \frac{\lambda(p-\alpha)(B-A)}{B} ; p+l+1 ; \frac{B}{B-1}\right)\right]^{-1} \\
& \quad-[\alpha \lambda+p(1-\lambda)+l]),
\end{aligned}
$$

when $A>-B[\alpha \lambda+p(1-\lambda)+l+1] / \lambda(p-\alpha)$. Further by taking $A \rightarrow(-B[\alpha \lambda+p(1-\lambda)+l+1] / \lambda(p-\alpha))^{+}$for the case
$A=-B[\alpha \lambda+p(1-\lambda)+l+1] / \lambda(p-\alpha)$ and using (78), we get (81). The result is the best possible as the function $q(z)$ is the best dominant of (78). This completes the proof of Theorem 15.

In the following section, we obtain the sufficient condition for the function $f$ to be a member of the class $\mathscr{R}_{p}^{m}(\lambda, l, \alpha)$.

\section{Sufficient Conditions for the Class $\mathscr{R}_{p}^{m}(\lambda, l, \alpha)$}

Theorem 16. If $f \in \mathscr{A}(p)$ satisfy the following subordination condition:

$$
(1-\beta) \frac{I_{p}^{m}(\lambda, l) f(z)}{z^{p}}+\beta \frac{I_{p}^{m+1}(\lambda, l) f(z)}{z^{p}} \prec 1+M_{1} z,
$$

where

$$
M_{1}=\frac{\lambda \beta(p-\alpha)(1+\lambda \beta /(p+l))}{|p+l-\lambda \beta(p-\alpha)|+\sqrt{(p+l)^{2}+(p+l+\lambda \beta)^{2}}},
$$

then $f \in \mathscr{R}_{p}^{m}(\lambda, l, \alpha)$.

Proof. Let

$$
g(z)=\frac{I_{p}^{m}(\lambda, l) f(z)}{z^{p}} .
$$

Then, the function $g$ is of the form (13) and is analytic in $\mathbb{U}$. From Theorem 12 with $A=M_{1}$ and $B=0$, we have

$$
g(z) \prec 1+\frac{p+l}{p+l+\lambda \beta} M_{1} z,
$$

which is equivalent to

$$
|g(z)-1|<\frac{p+l}{p+l+\lambda \beta} M_{1}=N<1 \quad(z \in \mathbb{U}) .
$$

If we set

$$
P(z)=\frac{1}{p-\alpha}\left(\frac{z\left(I_{p}^{m}(\lambda, l) f\right)^{\prime}(z)}{I_{p}^{m}(\lambda, l) f(z)}-\alpha\right) \quad(0 \leq \alpha<p) .
$$

Then, by using the identity (10) followed by (102), we obtain

$$
\frac{I_{p}^{m+1}(\lambda, l) f(z)}{z^{p}}=\left(1-\frac{\lambda(p-\alpha)}{p+l}+\frac{\lambda(p-\alpha)}{p+l} P(z)\right) g(z) .
$$

In view of (106), the hypothesis (100) can be written as follows:

$$
\left|\left(1-\frac{\lambda \beta(p-\alpha)}{p+l}\right) g(z)+\frac{\lambda \beta(p-\alpha)}{p+l} P(z) g(z)-1\right|<M_{1}
$$


We need to show that (107) yields

$$
\operatorname{Re} P(z)>0 \quad(z \in \mathbb{U}) .
$$

Suppose that this is false. Since $P(0)=1$, there exists a point $z_{0} \in \mathbb{U}$ such that $P\left(z_{0}\right)=i x$ for some $x \in \mathbb{R}$. Therefore, in order to show that (108), it is sufficient to obtain the contradiction from the inequality

$$
\begin{aligned}
E & =\left|\left(1-\frac{\lambda \beta(p-\alpha)}{p+l}\right) g\left(z_{0}\right)+\frac{\lambda \beta(p-\alpha)}{p+l} P\left(z_{0}\right) g\left(z_{0}\right)-1\right| \\
& \geq M_{1} .
\end{aligned}
$$

If we let $g\left(z_{0}\right)=u+i v$, then, by using (104) and the triangle inequality, we obtain that

$$
\begin{aligned}
E^{2}= & \mid\left(1-\frac{\lambda \beta(p-\alpha)}{p+l}\right) g\left(z_{0}\right) \\
& +\frac{\lambda \beta(p-\alpha)}{p+l} P\left(z_{0}\right) g\left(z_{0}\right)-\left.1\right|^{2} \\
= & \left(u^{2}+v^{2}\right)\left(\frac{\lambda \beta x(p-\alpha)}{p+l}\right)^{2}+\frac{2 v \lambda \beta x(p-\alpha)}{p+l} \\
& +\left|\left(1-\frac{\lambda \beta(p-\alpha)}{p+l}\right) g\left(z_{0}\right)-1\right|^{2} \\
\geq & \left(u^{2}+v^{2}\right)\left(\frac{\lambda \beta x(p-\alpha)}{p+l}\right)^{2}+\frac{2 v \lambda \beta x(p-\alpha)}{p+l} \\
& +\left(\frac{\lambda \beta(p-\alpha)}{p+l}-\left(1-\frac{\lambda \beta(p-\alpha)}{p+l}\right) N\right)^{2} .
\end{aligned}
$$

If we let

$$
\begin{aligned}
\Psi(x)= & E^{2}-M_{1}^{2} \\
\geq & \left(u^{2}+v^{2}\right)\left(\frac{\lambda \beta x(p-\alpha)}{p+l}\right)^{2}+\frac{2 v \lambda \beta x(p-\alpha)}{p+l} \\
& +\left(\frac{\lambda \beta(p-\alpha)}{p+l}-\left|1-\frac{\lambda \beta(p-\alpha)}{p+l}\right| N\right)^{2} \\
& -N^{2}\left(\frac{p+l+\lambda \beta}{p+l}\right)^{2},
\end{aligned}
$$

then (109) holds true if $\Psi(x) \geq 0$, for any $x \in \mathbb{U}$. Since $\left(u^{2}+\right.$ $\left.v^{2}\right)(\lambda \beta(p-\alpha) /(p+l))^{2}>0$, the inequality $\Psi(x) \geq 0$ holds true if the discriminant $\Delta \leq 0$; that is,

$$
\begin{gathered}
\Delta=4\left(\frac{\lambda \beta(p-\alpha)}{p+l}\right)^{2} \\
\times\left\{v^{2}-\left(u^{2}+v^{2}\right)\right. \\
\times\left[\left(\frac{\lambda \beta(p-\alpha)}{p+l}-\left|1-\frac{\lambda \beta(p-\alpha)}{p+l}\right| N\right)^{2}\right. \\
\left.\left.\quad-N^{2}\left(\frac{p+l+\lambda \beta}{p+l}\right)^{2}\right]\right\} \leq 0,
\end{gathered}
$$

which is equivalent to

$$
\begin{gathered}
v^{2}\left\{1-\left[\left(\frac{\lambda \beta(p-\alpha)}{p+l}-\left|1-\frac{\lambda \beta(p-\alpha)}{p+l}\right| N\right)^{2}\right.\right. \\
\left.\left.+N^{2}\left(\frac{p+l+\lambda \beta}{p+l}\right)^{2}\right]\right\} \\
\leq u^{2}\left[\left(\frac{\lambda \beta(p-\alpha)}{p+l}-\left|1-\frac{\lambda \beta(p-\alpha)}{p+l}\right| N\right)^{2}\right. \\
\left.-N^{2}\left(\frac{p+l+\lambda \beta}{p+l}\right)^{2}\right] .
\end{gathered}
$$

After a simple computation, by using (104), we obtain the inequality

$$
\begin{aligned}
\frac{v^{2}}{u^{2}} \leq & \frac{\rho^{2}}{1-\rho^{2}} \leq \frac{N^{2}}{1-N^{2}} \\
& \leq\left(\frac{\lambda \beta(p-\alpha)}{p+l}-\left|1-\frac{\lambda \beta(p-\alpha)}{p+l}\right| N\right)^{2} \\
& -N^{2}\left(\frac{p+l+\lambda \beta}{p+l}\right)^{2} \\
& \times\left(1-\left[\left(\frac{\lambda \beta(p-\alpha)}{p+l}-\left|1-\frac{\lambda \beta(p-\alpha)}{p+l}\right| N\right)^{2}\right.\right. \\
& \left.\left.\quad+N^{2}\left(\frac{p+l+\lambda \beta}{p+l}\right)^{2}\right]\right)^{-1},
\end{aligned}
$$

which yields $\Delta \leq 0$. Therefore $E \geq M_{1}$, which contradicts (107). It follows that $\operatorname{Re} P(z)>0$, and $f \in \mathscr{R}_{p}^{m}(\lambda, l, \alpha)$. 
Theorem 17. Let $\beta>0$ and if $f \in \mathscr{A}(p)$ such that $I_{p}^{m}(\lambda, l)$ $f(z) / z^{p} \neq 0, z \in \mathbb{U}$, satisfies the following differential subordination:

$$
\begin{aligned}
& (1-\beta)\left(\frac{I_{p}^{m}(\lambda, l) f(z)}{z^{p}}\right)^{\sigma} \\
& \quad+\beta \frac{\left(I_{p}^{m}(\lambda, l) f\right)^{\prime}(z)}{p z^{p-1}}\left(\frac{I_{p}^{m}(\lambda, l) f(z)}{z^{p}}\right)^{\sigma-1} \\
& \quad \prec 1+M_{2} z
\end{aligned}
$$

where the powers are understood as the principle value, and

$$
M_{2}= \begin{cases}\frac{(p-\alpha) \beta(1+\beta / \sigma p)}{|p-(p-\alpha) \beta|+\sqrt{p^{2}+(p+\beta / \sigma)^{2}}}, & \text { if } \sigma \neq 0, \\ \frac{(p-\alpha) \beta}{p}, & \text { if } \sigma=0,\end{cases}
$$

then $f \in \mathscr{R}_{p}^{m}(\lambda, l, \alpha)$.

Proof. If $\sigma=0$, then the condition (115) is equivalent to

$$
\left|\frac{z\left(I_{p}^{m}(\lambda, l) f\right)^{\prime}(z)}{I_{p}^{m}(\lambda, l) f(z)}-p\right|<p-\alpha \quad(z \in \mathbb{U}) .
$$

The above equation (117) implies that $f \in \mathscr{R}_{p}^{m}(\lambda, l, \alpha)$.

If we consider $\sigma>0$ and suppose that

$$
g(z)=\left(\frac{I_{p}^{m}(\lambda, l) f(z)}{z^{p}}\right)^{\sigma} \quad(z \in \mathbb{U}) .
$$

Choosing the principal value in (118), we note that $g$ is of the form (13) and is analytic in $\mathbb{U}$. Differentiating (118) with respect to $z$, we obtain

$$
\begin{aligned}
g(z)+ & \frac{\beta}{\sigma p} z g^{\prime}(z) \\
= & (1-\beta)\left(\frac{I_{p}^{m}(\lambda, l) f(z)}{z^{p}}\right)^{\sigma} \\
& +\beta \frac{\left(I_{p}^{m}(\lambda, l) f\right)^{\prime}(z)}{p z^{p-1}}\left(\frac{I_{p}^{m}(\lambda, l) f(z)}{z^{p}}\right)^{\sigma-1},
\end{aligned}
$$

which, in view of Lemma 2 with $c=\sigma p / \beta$, yields

$$
g(z) \prec 1+\frac{\sigma p}{\sigma p+\beta} M_{2} z \text {. }
$$

Also, with the aid of (118), (115) can be written as follows:

$$
g(z)\left\{1-\beta+\beta\left[\left(1-\frac{\alpha}{p}\right) P(z)+\frac{\alpha}{p}\right]\right\} \prec 1+M_{2} z
$$

where $P$ is given by (105). Therefore, by Lemma 8 , we find that

$$
\operatorname{Re} P(z)>0 \quad(z \in \mathbb{U})
$$

that is

$$
\operatorname{Re}\left(\frac{z\left(I_{p}^{m}(\lambda, l) f\right)^{\prime}(z)}{I_{p}^{m}(\lambda, l) f(z)}\right)>\alpha \quad(0 \leq \alpha<p, z \in \mathbb{U})
$$

which completes the proof of Theorem 17

By taking $m=0$ in Theorem 17, we get the following corollary due to Patel et al. [21].

Corollary 18. Let $\beta>0,0 \leq \alpha<p, p \in \mathbb{N}$, and $\sigma \geq 0$. If $f \in \mathscr{A}(p)$ such that $f(z) / z^{p} \neq 0(z \in \mathbb{U})$, and satisfies the following subordination condition:

$$
(1-\beta)\left(\frac{f(z)}{z^{p}}\right)^{\sigma}+\beta \frac{f^{\prime}(z)}{p z^{p-1}}\left(\frac{f(z)}{z^{p}}\right)^{\sigma-1} \prec 1+M_{2} z
$$

$(z \in \mathbb{U})$,

where the powers are understood as the principal value and $M_{2}$ is given by (116), then $f \in S_{p}^{*}(\alpha)$.

Remark 19. Taking $m=0$ and $p=1$ in Theorem 17, we get the result of Liu [20, Theorem 2.2, with $n=1$ ].

Remark 20. Putting $m=0$ and $\beta=p=1$ in Theorem 17, we obtain the result of Liu [20, Corollary 2.1, with $n=1$ ].

Remark 21. Putting $m=0$ and $p=\beta=\sigma=1$, in Theorem 17, we get the result of Mocanu and Oros [22, Corollary 2.2, with $n=1]$.

Remark 22. Taking $m=\alpha=0$, and $p=\beta=\sigma=1$ in Theorem 17, we obtain the result of Mocanu [23, with $n=1$ ].

Taking $m=0$ and $\beta=1 /(p-\alpha), 0 \leq \alpha<p$ in Theorem 17, we obtain the following corollary.

Corollary 23. Let $\sigma>0,0 \leq \alpha<p$, and $p \in \mathbb{N}$. If $f \in \mathscr{A}(p)$ such that $f(z) / z^{p} \neq 0(z \in \mathbb{U})$ satisfies the inequality:

$$
\begin{aligned}
& \left|(p-\alpha-1)\left(\frac{f(z)}{z^{p}}\right)^{\sigma}+\frac{f^{\prime}(z)}{p z^{p-1}}\left(\frac{f(z)}{z^{p}}\right)^{\sigma-1}+\alpha-p\right| \\
& <\frac{((p-\alpha) / p)[p \sigma(p-\alpha)+1]}{\sigma(p-\alpha)|p-1|+\sqrt{[p \sigma(p-\alpha)]^{2}+[p \sigma(p-\alpha)+1]^{2}}}
\end{aligned}
$$

where the powers are the principal value ones, then $f \in S_{p}^{*}(\alpha)$. Remark 24. Taking $p=1$ in Corollary 23, we obtain the result of Liu [20, Corollary 2.2, with $n=1]$. 
Remark 25. Putting $p=\sigma=1$ in Corollary 23, we get the result of Mocanu and Oros [22, Corollary 2.4, with $n=1$ ].

In Theorem 26, we obtain the necessary condition for functions belonging to class $P\left(\gamma_{j}\right)$.

Theorem 26. Let $f_{j} \in \mathscr{A}(p)(j=1,2)$. If the functions $I_{p}^{m+1}(\lambda, l) f_{j}(z) / z^{p} \in P\left(\gamma_{j}\right)\left(0 \leq \gamma_{j}<1\right)$, then the function $g=I_{p}^{m}(\lambda, l),\left(f_{1} * f_{2}\right)$ satisfies the following inequality:

$$
\operatorname{Re}\left(\frac{I_{p}^{m+1}(\lambda, l) g(z)}{I_{p}^{m}(\lambda, l) g(z)}\right)>0 \quad(z \in \mathbb{U})
$$

provided that

$$
\begin{aligned}
& 2\left(1-\gamma_{1}\right)\left(1-\gamma_{2}\right) \\
& \quad<\frac{\lambda+2(p+l)}{\lambda\left[{ }_{2} F_{1}(1,1 ;(p+l) / \lambda+1 ; 1 / 2)-1\right]^{2}+2(p+l)} .
\end{aligned}
$$

Proof. Let $g=I_{p}^{m}(\lambda, l)\left(f_{1} * f_{2}\right)$. By using Lemma 4 and (10), we obtain

$$
\begin{aligned}
& \operatorname{Re}\left(\frac{I_{p}^{m+1}(\lambda, l) f_{1}(z)}{z^{p}} * \frac{I_{p}^{m+1}(\lambda, l) f_{2}(z)}{z^{p}}\right) \\
& \quad=\operatorname{Re}\left(\frac{I_{p}^{m+1}(\lambda, l) g(z)}{z^{p}}+\frac{\lambda z}{p+l}\left(\frac{I_{p}^{m+1}(\lambda, l) g(z)}{z^{p}}\right)^{\prime}\right) \\
& \quad>1-2\left(1-\gamma_{1}\right)\left(1-\gamma_{2}\right) .
\end{aligned}
$$

Then, by using Lemma 2 with $c=(p+l) / \lambda, A=-1+4(1-$ $\left.\gamma_{1}\right)\left(1-\gamma_{2}\right)$, and $B=-1$, we have

$$
\begin{aligned}
\operatorname{Re}\left(\frac{I_{p}^{m+1}(\lambda, l) g(z)}{z^{p}}\right)> & 1+2\left(1-\gamma_{1}\right)\left(1-\gamma_{2}\right) \\
& \times\left[{ }_{2} F_{1}\left(1,1 ; \frac{p+l}{\lambda}+1 ; \frac{1}{2}\right)-1\right] .
\end{aligned}
$$

Again, from (129) and Corollary 13, we obtain

$$
\begin{aligned}
\operatorname{Re}\left(\frac{I_{p}^{m}(\lambda, l) g(z)}{z^{p}}\right)> & 1-2\left(1-\gamma_{1}\right)\left(1-\gamma_{2}\right) \\
& \times\left[{ }_{2} F_{1}\left(1,1 ; \frac{p+l}{\lambda}+1 ; \frac{1}{2}\right)-1\right]^{2}
\end{aligned}
$$

Now, if we let $\varphi(z)=I_{p}^{m+1}(\lambda, l) g(z) / I_{p}^{m}(\lambda, l) g(z)(z \in \mathbb{U})$ then $\varphi$ is of the form (13) and is analytic in $\mathbb{U}$. A simple computation shows that

$$
\begin{aligned}
& \frac{I_{p}^{m+1}(\lambda, l) g(z)}{z^{p}}+\frac{\lambda z}{p+l}\left(\frac{I_{p}^{m+1}(\lambda, l) g(z)}{z^{p}}\right)^{\prime} \\
& \quad=\frac{I_{p}^{m}(\lambda, l) g(z)}{z^{p}}\left[\varphi^{2}(z)+\frac{z \lambda \varphi^{\prime}(z)}{p+l}\right] \\
& \quad=\Psi\left(\varphi(z), z \varphi^{\prime}(z) ; z\right),
\end{aligned}
$$

where $\Psi(u, v ; z)=\left(I_{p}^{m}(\lambda, l) g(z) / z^{p}\right)\left(u^{2}+\lambda v /(p+l)\right)$.

Thus, by using (128), (131) can be written as follows:

$$
\operatorname{Re}\left(\Psi\left(\varphi(z), z \varphi^{\prime}(z) ; z\right)\right)>1-2\left(1-\gamma_{1}\right)\left(1-\gamma_{2}\right) .
$$

For all real $x$ and $y \leq-(1 / 2)\left(1+x^{2}\right)$, we have

$$
\begin{aligned}
\operatorname{Re} & (\Psi(i x, y ; z)) \\
= & \left(\frac{\lambda y}{p+l}-x^{2}\right) \operatorname{Re}\left(\frac{I_{p}^{m}(\lambda, l) g(z)}{z^{p}}\right) \\
\leq & -\frac{\lambda}{2(p+l)}\left[1+\left(1+\frac{2(p+l)}{\lambda}\right) x^{2}\right] \\
& \times \operatorname{Re}\left(\frac{I_{p}^{m}(\lambda, l) g(z)}{z^{p}}\right) \\
\leq & -\frac{\lambda}{2(p+l)} \operatorname{Re}\left(\frac{I_{p}^{m}(\lambda, l) g(z)}{z^{p}}\right) \\
< & 1-2\left(1-\gamma_{1}\right)\left(1-\gamma_{2}\right) \quad(z \in \mathbb{U}),
\end{aligned}
$$

where we used (127) and (130). Thus, by Lemma 9, we get

$$
\operatorname{Re}(\varphi(z))>0 \quad(z \in \mathbb{U}),
$$

which completes the proof of Theorem 26.

\section{Conflict of Interests}

The authors declare that there is no conflict of interests regarding the publication of this paper.

\section{Acknowledgment}

The authors thank the referees for their insightful suggestions.

\section{References}

[1] S. S. Miller and P. T. Mocanu, "Differential subordinations and univalent functions," The Michigan Mathematical Journal, vol. 28, no. 2, pp. 157-172, 1981.

[2] S. S. Miller and P. T. Mocanu, Differential Subordinations: Theory and Applications, vol. 225 of Monographs and Textbooks in Pure and Applied Mathematics, Marcel Dekker, New York, NY, USA, 2000. 
[3] A. Cătaş, "On certain classes of $p$-valent functions defined by multiplier transformations," in Proceedings of the International Symposium on Geometric Function Theory and Applications (GFTA '07), S. Owa and Y. Polatoglu, Eds., vol. 91, pp. 241-250, TC İstanbul Kültür University Publications, Istanbul, Turkey, August 2007.

[4] H. M. Srivastava, K. Suchithra, B. A. Stephen, and S. Sivasubramanian, "Inclusion and neighborhood properties of certain subclasses of analytic and multivalent functions of complex order," Journal of Inequalities in Pure and Applied Mathematics, vol. 7, no. 5, pp. 1-8, 2006.

[5] S. Sivaprasad Kumar, H. C. Taneja, and V. Ravichandran, "Classes of multivalent functions defined by Dziok-Srivastava linear operator and multiplier transformation," Kyungpook Mathematical Journal, vol. 46, no. 1, pp. 97-109, 2006.

[6] N. E. Cho and T. H. Kim, "Multiplier transformations and strongly close-to-convex functions," Bulletin of the Korean Mathematical Society, vol. 40, no. 3, pp. 399-410, 2003.

[7] N. E. Cho and H. M. Srivastava, "Argument estimates of certain analytic functions defined by a class of multiplier transformations," Mathematical and Computer Modelling, vol. 37, no. 1-2, pp. 39-49, 2003.

[8] M. Kamali and H. Orhan, "On a subclass of certain starlike functions with negative coefficients," Bulletin of the Korean Mathematical Society, vol. 41, no. 1, pp. 53-71, 2004.

[9] H. Orhan and H. Kiziltunç, "A generalization on subfamily of p-valent functions with negative coefficients," Applied Mathematics and Computation, vol. 155, no. 2, pp. 521-530, 2004.

[10] G. S. Sălăgean, "Subclasses of univalent functions," in Complex Analysis-Fifth Romanian-Finnish Seminar, vol. 1013 of Lecture Notes in Mathematics, pp. 362-372, Springer, Berlin, Germany, 1983.

[11] F. M. Al-Oboudi, "On univalent function defined by a generalized Sălăgean operator," International Journal of Mathematics and Mathematical Sciences, vol. 27, pp. 1429-1436, 2004.

[12] B. A. Uralegaddi and C. Somanatha, "Certain classes of univalent functions," in Current Topics in Analytic Function Theory, pp. 371-374, World Scientific, Singapore, 1992.

[13] T. Bulboacă, M. K. Aouf, and R. M. El-Ashwah, "Subordination properties of multivalent functions defined by certain integral operetor," Banach Journal of Mathematical Analysis, vol. 6, no. 2, pp. 69-85, 2012.

[14] J. Patel and A. K. Mishra, "On certain subclasses of multivalent functions associated with an extended fractional differintegral operator," Journal of Mathematical Analysis and Applications, vol. 332, no. 1, pp. 109-122, 2007.

[15] D. Z. Pashkouleva, "The starlikeness and spiral-convexity of certain subclasses of analytic functions," in Current Topics in Analytic Function Theory, pp. 266-273, World Scientific, Singapore, 1992.

[16] J. Stankiewicz and Z. Stankiewicz, "Some applications of the Hadamard convolution in the theory of functions," Annales Universitatis Mariae Curie-Skłodowska A, vol. 40, pp. 251-265, 1986.

[17] E. T. Whittaker and G. N. Watson, A Course of Modern Analysis: An Introduction to the General Theory of Infinite Processes and of Analytic Functions, Cambridge Mathematical Library, Cambridge University Press, Cambridge, UK, 1996.

[18] S. S. Miller and P. T. Mocanu, "Univalent solutions of BriotBouquet differential equations," Journal of Differential Equations, vol. 56, no. 3, pp. 297-309, 1985.
[19] D. R. Wilken and J. Feng, "A remark on convex and starlike functions," The Journal of the London Mathematical Society, vol. 21, no. 2, pp. 287-290, 1980.

[20] M. Liu, "On certain sufficient condition for starlike functions," Soochow Journal of Mathematics, vol. 29, no. 4, pp. 407-412, 2003.

[21] J. Patel, A. K. Mishra, and H. M. Srivastava, "Classes of multivalent analytic functions involving the Dziok-Srivastava operator," Computers \& Mathematics with Applications, vol. 54, no. 5, pp. 599-616, 2007.

[22] P. T. Mocanu and Gh. Oros, "A sufficient condition for starlikeness of order $\alpha$," International Journal of Mathematics and Mathematical Sciences, vol. 28, no. 9, pp. 557-560, 2001.

[23] P. T. Mocanu, "Some simple criteria for starlikeness and convexity," Libertas Mathematica, vol. 13, pp. 27-40, 1993. 


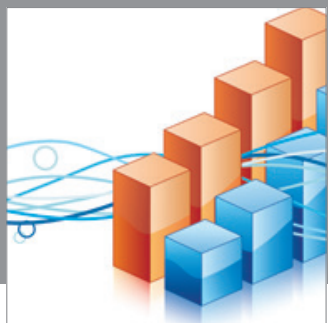

Advances in

Operations Research

mansans

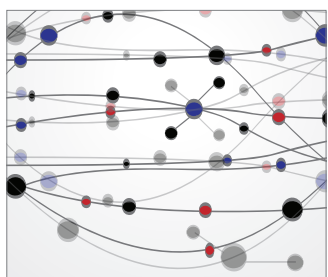

The Scientific World Journal
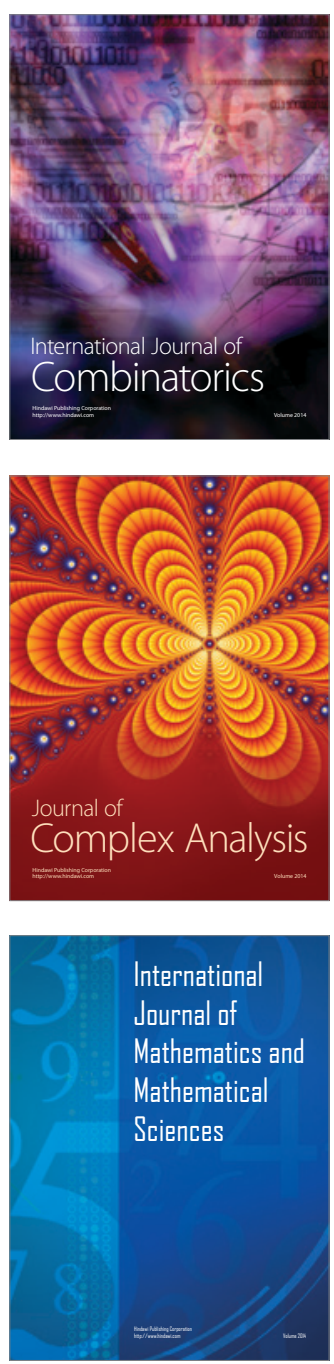
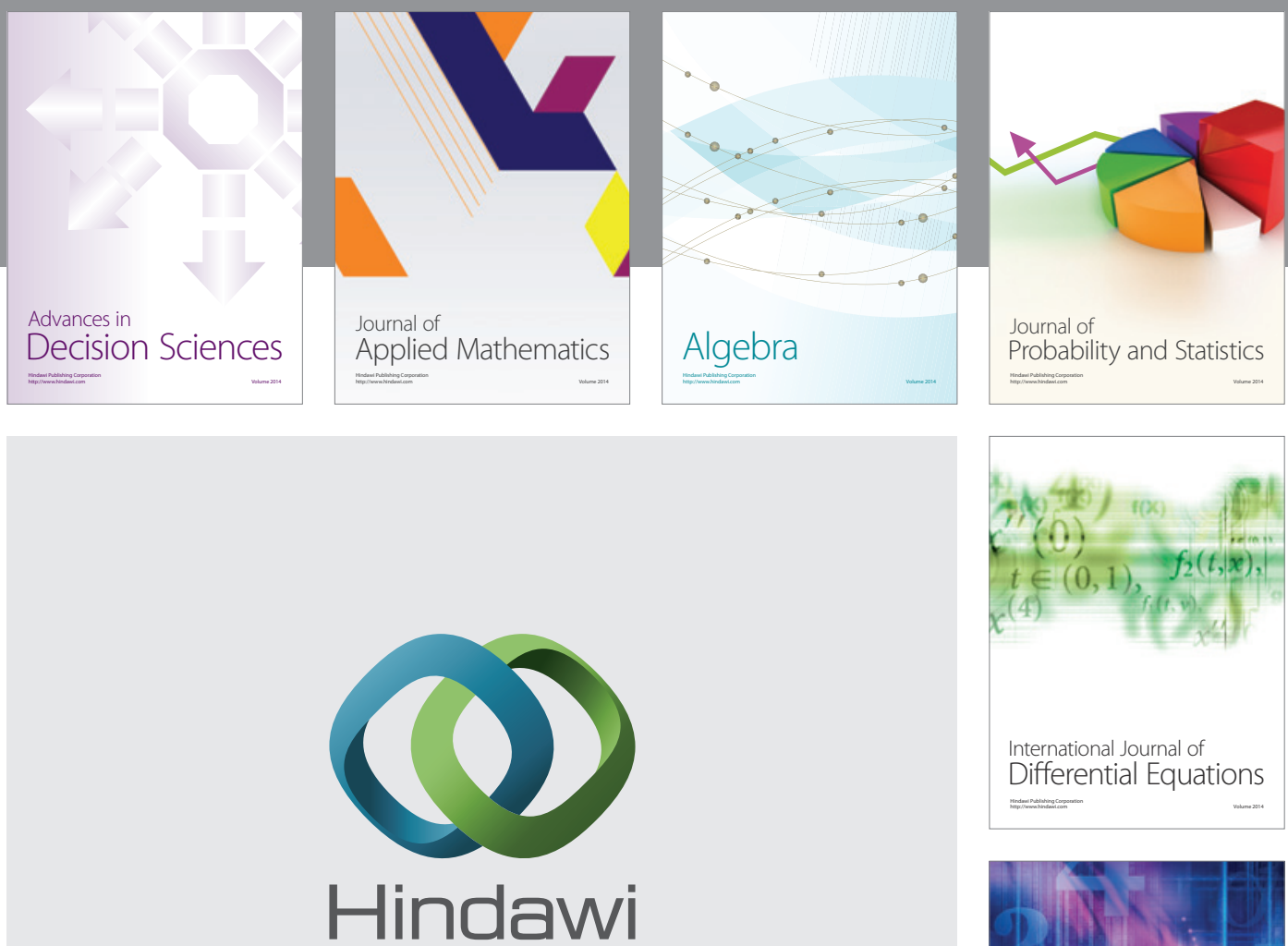

Submit your manuscripts at http://www.hindawi.com
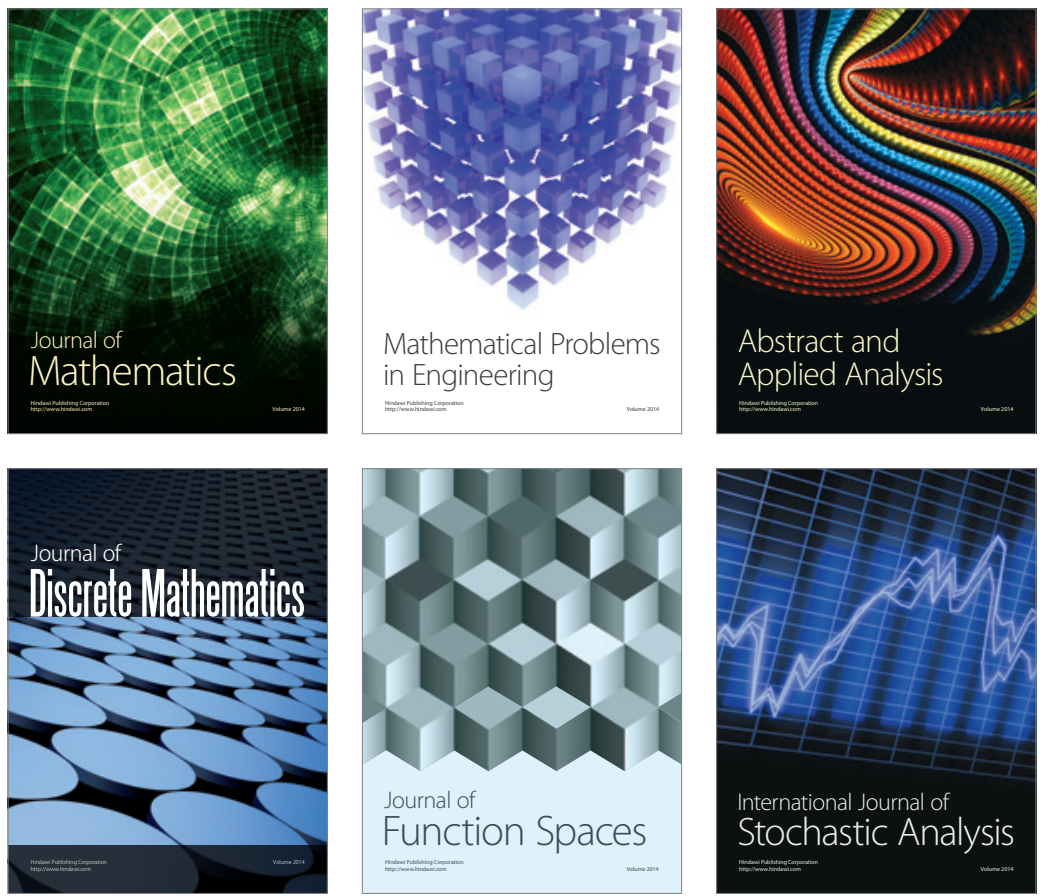

Journal of

Function Spaces

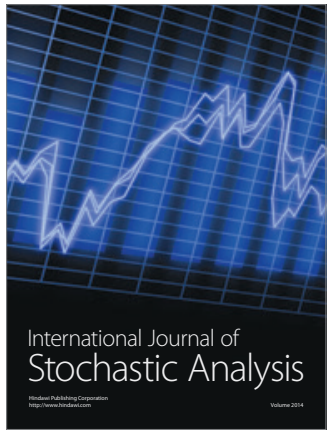

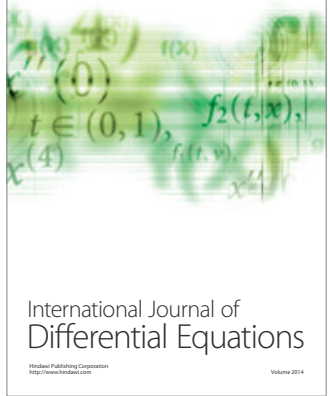
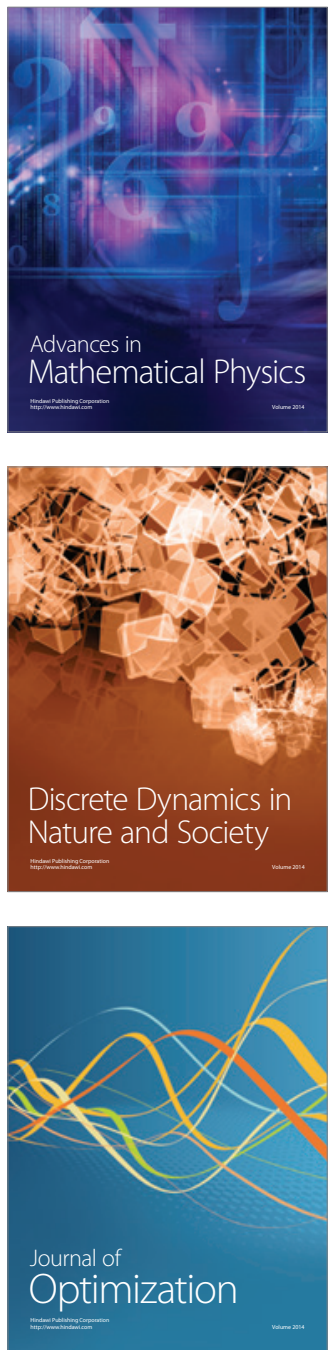